\title{
Interaction of small aromatic molecules: An ab initio studies on benzene and pyridine molecules
}

\author{
Bipul Bezbaruah, P. Hazarika, A. Gogoi, O.K. Medhi, C. Medhi*
}

Chemistry Department, Gauhati University, Guwahati, Assam, India; *Corresponding Author: chitrani@sify.com

Received 10 September 2010; revised 1 November 2010; accepted 22 November 2010.

\begin{abstract}
The use of appropriate level of theories for studying weak interactions such as stacking of aromatic molecules has been an important aspect, since the high level methods have limitations for application to large molecules. The differences in the stacking energies of various structures are found significant for identifying the most favored stacked benzene rings and the pyridine rings. The most favored structure of benzene rings obtained from various methods are similar, and also comparable with that of reported accurate $\operatorname{CCSD}(\mathrm{T})$ method. The effect of basis set in the stacking energies of MP2 calculations is small. Thus the moderately accurate methods may be feasible for studying the stacking interactions as demonstrated for benzene and pyridine molecules.
\end{abstract}

Keywords: Ab Initio; Stacking; Basis Set; Benzene; Pyridine

\section{INTRODUCTION}

The non bonded interactions between aromatic compounds are considered to be an important aspect in biological systems, and other relevant areas like drug discovery. These attractive intermolecular interactions are also responsible for macromolecular aggregation, where the medicinal property of a drug molecule may partly depend on its recognition for a biological system [1-4]. The majority of medicinal agents contain aromatic substituents that provide one of the important groups related to medicinal property. For example, the biological activity of many anticancer drugs, Amsacrine and Daunomycin is believed to be related to the drug intercalation within sequences of DNA, where the stacking interactions between aromatic rings and base pairs usually take place [1-4]. As a part of designing new intercalative drugs, the stacking interactions between the aromatic rings have been analyzed to achieve the sufficient struc- tural requirement of the DNA binding domain of drugs. So it is rather important to choose the most reliable method to understand the structure and non-bonded interactions of aromatic molecules. We note that the benzene and the pyridine rings are the basic molecular parts that constitute most biomolecules and drugs. At the first sight, the stacking interactions of benzene-benzene and pyridine-pyridine could represent the constituent units for the stabilization of many large aromatic molecules. So the studies on small aromatic molecules may be given particular importance for better understanding of stacking interactions among large aromatic molecules.

$A b$ initio calculations have been shown useful for studying the non bonded interactions of aromatic molecules [5-10]. The various levels of theories and basis sets have been carefully chosen to calculate the interaction energies of aromatic $(\pi-\pi)$ and stacking types of interactions that are responsible for the stabilization of double helical DNA. It is evident that the studies of molecular stacking require high level $a b$ initio calculations. Many theoretical studies are reported to predict the preferred stacking interactions of benzene dimers, and thereby provoking the importance of dipole-dipole and quadrupole-quadrupole interaction [5-6]. Similar studies on the intermolecular interactions of cytosine dimers have been calculated with MP2/6-31G** level, whereas less accurate approach, the AMBER force field is also found useful in certain cases despite of the lack of estimating dispersion energies in the calculations. The dispersion forces, the short range exchange repulsions and electron correlation are the essential factors for the overall assessment of stacking interaction of molecules [7-10]. Comparison of different level of theories used in certain investigations clearly indicate the choice of reasonably accurate method that include proper basis set as well as electron correlation in the calculations. As we know that the London dispersion force is the instrumental binding of stacked molecules, which is not described by Hartree-Fock theory. Therefore, the correlated descriptions of electrons are must to produce even a qualitatively accurate picture of weakly interacted molecules. It 
should be noted that several newly developed functional have shown to provide acceptable results for this type of intermolecular interactions [10-13].

However, this method is still rather expensive for large molecules, and it can only be applied to small molecules such as benzene dimers. The $\operatorname{CCSD}(\mathrm{T})$ method is currently believed to be the most accurate for computing dispersion interactions provided the basis set of aug-cc-pVTZ is included in the calculations [9-14]. The computational cost required for larger molecules does not permit the application of accurate $a b$ initio methods. After thorough survey of the available studies on the molecular stacking of small aromatic rings, there are certain issues that need to be reinvestigated. The present investigation aims to test the different level of $a b$ initio methods in predicting the stability of benzene and pyridine dimers [15].

\section{METHODOLOGY}

The completely optimized geometries of benzene and pyridine molecules with $\mathrm{HF} / 6-31 \mathrm{G}^{* *}$ were taken for calculating stacking energies. Single point calculations on the stacked models have been carried out with MP2 as well as HF methods with some chosen basis set. The two benzene rings may stack either in eclipsed (exact $\pi-\pi$ sandwiched form) or in staggered configuration (Figure 1a). All the stacked configurations of benzene rings have been analyzed by rotating the upper benzene through different angles about the vertical axis at fixed vertical separation (optimum distance of $3.3 \AA$ ). The

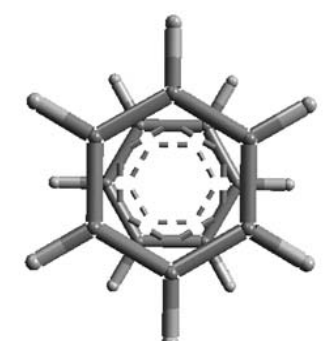

(a)

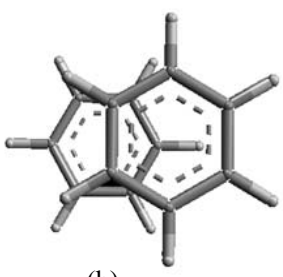

(b)

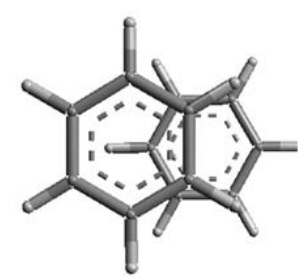

(c)
Figure 1. (a) Staggered model of two stacked benzene molecules, (b) Optimum stacked structure for lateral shifting along positive axes with origin $(0,0)$ for complete stacking, and (c) Optimum stacked structure for lateral shifting along negative axes with origin $(0,0)$ for complete stacking. single point calculations have been performed for all the structures. The eclipsed form has been taken for constructing various stacked models by shifting one benzene ring laterally along the plane of the other, and the most favorable structures are shown in Figures 1b and 1c.

The interaction energies are computed from the following equation.

$$
\text { Interaction energies }=\mathrm{E}_{\mathrm{ST}}-2 \mathrm{E}_{\mathrm{M}}
$$

$\mathrm{E}_{\mathrm{ST}}$ and $\mathrm{E}_{\mathrm{M}}$ are the energies of stacked model and monomer. All the calculations are carried out with Gaussian 03 program code [15].

\section{RESULTS AND DISCUSSION}

The relative changes of the stacking energies (MP2) of different stacked benzene rings are shown in Figure 2, and certain stable structures are located from the local minima in the potential energy plots. However the corresponding potential energy plots of HF calculations shown in Figure 3 cannot explain the stacking stabilization of two benzene rings. The single point calculations of all stacked models have been performed in this study, since the complete geometry optimization may not be advantageous to locate the local optimum structures. The nature of the potential energy plots of MP2 and HF calculations shown in Figures $\mathbf{2}$ and $\mathbf{3}$ is similar, but the HF calculation fail to locate the local minima for stable structures. The values are found significantly different, and the MP2/6-31+G(d,p) and MP2/6-31+G(df,p) calculations could estimate large negative interaction energies compared to that of HF calculations.

It is not surprising that the interaction energies of $\mathrm{HF} / 6-31 \mathrm{G}^{* *}$ and $\mathrm{HF} / 6-31+\mathrm{G}(\mathrm{d}, \mathrm{p})$ calculations are all positive, which is definitely due to the lack of dispersion energies with these calculations. The series of results could provide prior necessity of dispersion forces for the stabilization of these stacked molecules. The results of HF and MP2 level of theories reflect the extent of dispersion energies accounted in all these calculations. Indeed, the electron correlations included in MP2 level with diffused function in the basis set could estimate more negative stacking energies, where the increase of diffuse function in the basis set provides little change in the energy values.

As we know that the stacking energies obtained from HF calculation include columbic, induction, exchange and some electron correlation energies, and the intermolecular electron correlation necessary for the stabilization of these stacked molecules cannot be calculated with this method. However the stacking energies obtained from this method may be taken for comparison with the MP2 results. The present studies focus how the stacking energies can be improved with the inclusion of 


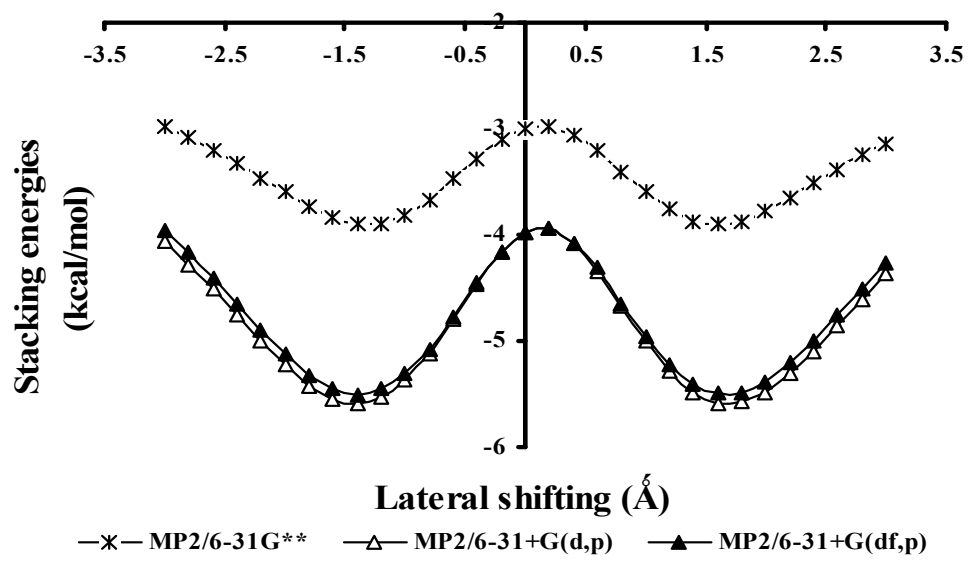

Figure 2. Plots of benzene-benzene stacking energies versus lateral shifting in angstrom with different basis sets in the MP2 method (for benzene-benzene staggered models).

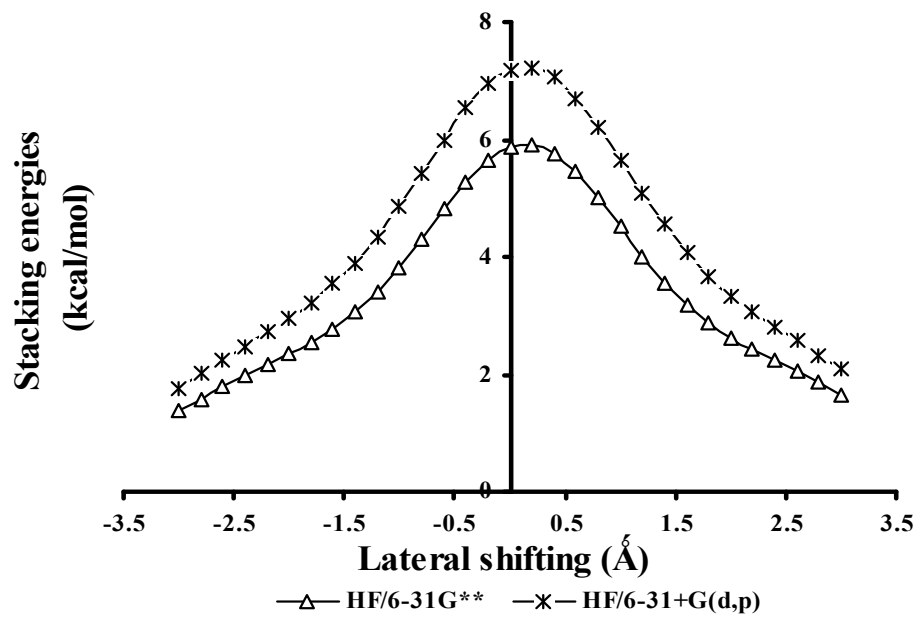

Figure 3. Plots of benzene-benzene stacking energies versus lateral shifting in angstrom with different basis sets in the HF method (for benzene-benzene staggered models).

Table 1. Computed stacking energies of benzene-benzene and pyridine-pyridine rings for optimized stacked models with different basis sets.

\begin{tabular}{|c|c|c|c|c|}
\hline \multirow{3}{*}{ Basis sets } & \multicolumn{4}{|c|}{ Stacking energies $(\mathrm{kcal} / \mathrm{mol})$} \\
\hline & \multicolumn{2}{|c|}{ Benzene-Benzene } & \multicolumn{2}{|c|}{ Pyridine-Pyridine } \\
\hline & Eclipsed & Staggered & Eclipsed & Staggered \\
\hline $\mathrm{HF} / 6-31 \mathrm{G} * *$ & 2.174 & 3.051 & 1.611 & 2.886 \\
\hline $\mathrm{HF} / 6-31+\mathrm{G}(\mathrm{d}, \mathrm{p})$ & 3.318 & 3.105 & 2.571 & 3.515 \\
\hline $\mathrm{MP} 2 / 6-31 \mathrm{G}^{* *}$ & -3.149 & -3.898 & -3.075 & -6.236 \\
\hline MP2/6-31+G(d,p) & -5.447 & -5.594 & -4.971 & -6.448 \\
\hline MP2/6-31+G(df,p) & -4.591 & -5.506 & -3.258 & -6.462 \\
\hline
\end{tabular}

diffused functions in the HF and MP2 level of calculations. The computed stacking energies of optimum structures with various levels of calculations are summarized in Table 1.

It may be noted that the difference of stacking energies obtained from $\mathrm{HF} / 6-31 \mathrm{G}^{* *}$ and $\mathrm{MP} 2 / 6-31 \mathrm{G}^{* *}$ calculations is significantly large, whereas values of
MP2/6-31G**, MP2/6-31+G(d,p) and MP2/6-31+G(df,p) are not so different (Table 1). However the most expensive method, MP4 level of theory is particularly used in most calculations on stacking interactions, but such high level calculations cannot be applied to large molecule of research interests. For general application one must test the low level methods as well to extract some informa- 


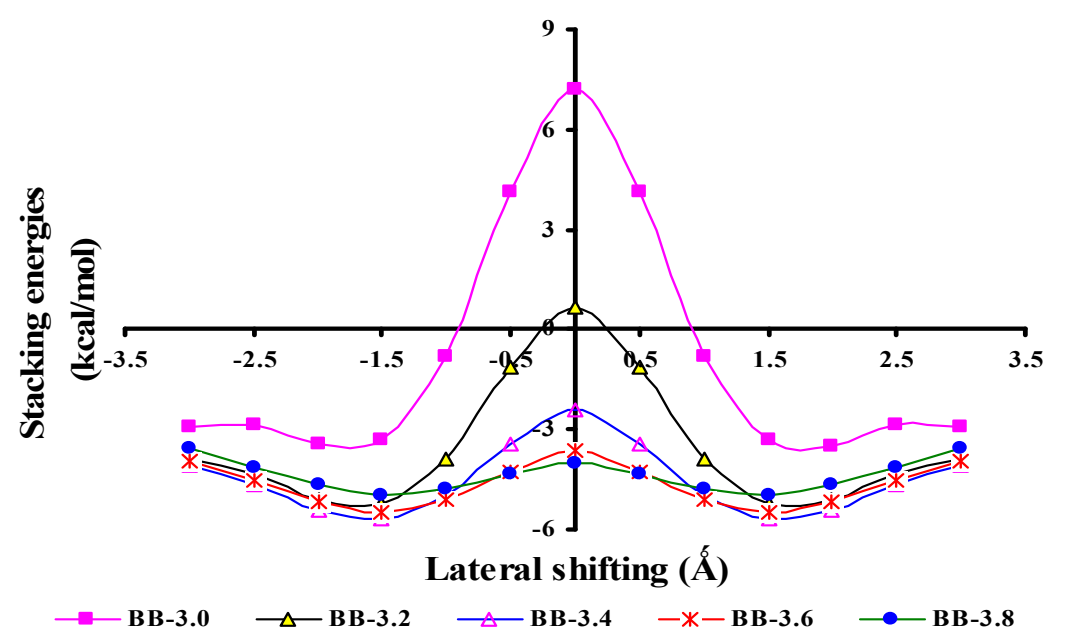

Figure 4. Plots of stacking energies versus lateral shifting in angstrom $(\AA)$ of benzene molecules at different intermolecular distances with MP2/6-31+G(d,p)

tion. The change in stacking energies determined by MP2 method with $6-31+\mathrm{G}(\mathrm{d}, \mathrm{p})$ and $6-31+\mathrm{G}(\mathrm{df}, \mathrm{p})$ may be appropriate for qualitative explanations of these non-bonded weak interactions. Our results show that the stacking energies do not considerably vary with the inclusion of more diffuse functions in the basis set. The stacking energies obtained from MP2/6-31+G(d,p) calculations are found much better than the reported values of MP2 and CCSD(T) methods with aug-cc-pVQZ and aug-cc-pVDZ basis set [7,9]. The reported $\operatorname{CCSD}(\mathrm{T})$ energy is $-2.72 \mathrm{kcal} / \mathrm{mol}$ with basis set correction, whereas the values for MP2/6-31+G(d,p) and MP2 $/ 6-31+\mathrm{G}(\mathrm{df}, \mathrm{p})$ without basis set correction are $-5.447 \mathrm{kcal} / \mathrm{mol}$ and $-4.591 \mathrm{kcal} / \mathrm{mol}$. The single-point MP2 calculations with $6-31+\mathrm{G}(\mathrm{d}, \mathrm{p})$ basis set have been found useful in describing the stability of stacked benzene and pyridine molecules. The calculated interaction energies with $6-31+\mathrm{G}(\mathrm{df}, \mathrm{p})$ basis set does not show much variation from that of $6-31+G(d, p)$ basis set (Table 1, Figure 2). The basis set superposition error at the MP2 level is not so essential although there may be slight effect on the dispersion energies.

Although the HF and other low level methods cannot be used to calculate accurate stacking energies, it may be useful for predicting at least the stabilization of counter molecules qualitatively. As we can see that both the HF and MP2 methods can locate similar regions for the stabilization of stacked structures (Figures 2 and 3).

It is also important to locate the most favored $\pi-\pi$ stacking distance, so the stacking energies of two benzene molecules at different vertical separations have been calculated and the distance(vertical and lateral) dependent variation of stacking energies are shown in Figure 4. The stacked structures located within certain conformational spaces of two benzene are found favorable as predicted from the results of MP2/6-31G(d,p) calculations. The maxima and minima in the curves indicate the two distinguishable regions of total and partial stacking of aromatic rings(Figure 2, Figures 1(a-c)). The repulsive interaction is found prominent in the total stacking of the aromatic rings, and the maximum points in the curves represent the unstable structures. Also the repulsive interaction is found maximum at $3.0 \AA$, and gradually decreases due to the increase of electrostatic interaction energies at longer distances.

As shown in Table 1, the extent of dispersion energies included in the stacking energies of MP2/6-31G**, $\mathrm{MP} 2 / 6-31+\mathrm{G}(\mathrm{d}, \mathrm{p})$ and $\mathrm{MP} 2 / 6-31+\mathrm{G}(\mathrm{df}, \mathrm{p})$ calculations of benzene and pyridine molecules is distinct, whereas the $\mathrm{HF} / 6-31 \mathrm{G}^{* *}$ calculation cannot usually estimate dispersion energies.

\section{CONCLUSIONS}

The extent of dispersion forces included in various calculations may be useful for monitoring relative variation of stacking interactions of aromatic molecules. The results of MP2 studies and the reported CCSD(T) calculations can provide similar information on the stacking of benzene rings. Hence the MP2/6-31G+(d,p) and MP2/6-31+G(df,p) may be feasible for explaining the $\pi-\pi$ type of stacking interaction qualitatively, and the method may be applied to the computation of large stacked molecules instead of other high level expensive techniques.

\section{ACKNOWLEDGMENTS}

The authors thank Department of Science and Technology and Council of Scientific Research, New Delhi, India, for financial assistance. 


\section{REFERENCES}

[1] Castonguay, L.A., Rappe', A.K. and Casewit, C.J. (1991) $\Pi$-stacking and the platinum-catalyzed asymmetric hydroformylation reaction: A molecular modeling study. Journal of the American Chemical Society, 113(19), 7177-7183.

doi:10.1021/ja00019a013

[2] Kolb, H.C., Andersson, P.G. and Sharpless, K.B. (1994) Toward an understanding of the high enantioselectivity in the osmium-catalyzed asymmetric dihydroxylation (AD). 1. Kinetics. Journal of the American Chemical Society, 116(4), 1278-1291. doi: $10.1021 / \mathrm{ja} 00083 \mathrm{a} 014$

[3] Smith, D.A., Ulmer II, C.W., and Gilbert M.J. (1992) Structural studies of aromatic amines and the dna intercalating compounds $m$-amsa and $O$-amsa: Comparison of mndo, am1, and pm3 to experimental and ab initio results. Journal of Computational Chemistry, 13 (5), 640. doi: $10.1002 /$ jcc. 540130514

[4] Barone, G., Guerra, C.F., Gambino, N., Silvestri, A., Lauria, A., Almerico, A.M. and Bickelhaupt, F.M. (2008) Intercalation of daunomycin into stacked DNA base pairs. DFT study of an anticancer drug. Journal of Biomolecular Structure and Dynamics, 26(1), 115-30.

[5] Gilman, A.G., Rall, T.W., Mies, A.S. and Taylor, P. (1993) The pharmaceutical basis of therapeutics. 8th Ed., McGraw Hill, Inc., New York.

[6] Ishida, T., Doi, M., Ueda, H., Inoue, M. and Scheldrick, G.M. (1988) Specific ring stacking interaction on the tryptophan-7-methylguanine system: Comparative crystallographic studies of indole derivatives-7-methylguanine base, nucleoside, and nucleotide complexes. Journal of the American Chemical Society, 110 (7), 2286-2294 doi:10.1021/ja00215a046

[7] Hobza, P., Selzle, H.L. and Schlag, E.W. (1994) Potential energy surface of the benzene dimer: Ab initio theoretical study. Journal of the American Chemical society, 116(8), 3500-3506.

doi:10.1021/ja00087a041

[8] Bernstein, E.R., Sun, S. (1996) Aromatic van der waals clusters: Structure and nonrigidity. Journal of Physical Chemistry, 100(32), 13348-13366. doi:10.1021/jp960739o
[9] Hobza, P., Selzle, H.L., Schlag, E.W. (1996) Potential energy surface for the benzene dimer. Results of $a b$ initio $\operatorname{CCSD}(\mathrm{T})$ calculations show two nearly isoenergetic structures: T-Shaped and parallel-displaced. Journal of Physical Chemistry, 100(48), 18790. doi:10.1021/jp961239y

[10] Tsuzuki, S., Honda, K., Uchimaru, T., Mikami, M. and Tanabe, K. (2002) Origin of attraction and directionality of the $\pi-\pi$ interaction: Model chemistry calculations of benzene dimer interaction. Journal of the American Chemical Society, 124(1), 104-112. doi:10.1021/ja0105212

[11] Sinnokrot, M.O., Valeev, E. F. and Sherrill, C.D. (2002), Estimates of the $a b$ initio limit for $\pi-\pi$ interactions: The benzene dimer. Journal of the American Chemical Society, 124(36), 10887-10893. doi:10.1021/ja025896h

[12] Zhikol, O.A., Shishkin, O.V., Lyssenko, K.A. and Leszczynski, J. (2005) Electron density distribution in stacked benzene dimers: A new approach towards the estimation of stacking interaction energies. Journal of Chemical Physics, 122(14), 144104. doi:10.1063/1.1877092

[13] Parthasarathi, R., Subramanian, V. (2005) Stacking interactions in benzene and cytosine dimers: From molecular electron density perspective. Structural Chemistry, 16(3), 243-255. doi:10.1007/s11224-005-4455-8

[14] Steven, E.W., Anne, J.M, Peter, M, Timothy, M.S, and Houk, K.N. (2010) Probing substituent effects in aryl-aryl interactions using stereoselective diels-alder cycloadditions. Journal of the American Chemical Society, 132(10), 3304-3311. doi: $10.1021 / \mathrm{ja} 903653 \mathrm{j}$

[15] Frisch, M.J., Trucks, G.W., Schlegel, H.B., Gill, P.M.W., Johnson, B.G., Robb, M.A., Cheeseman, J.R., Keith, T., Petersson, G.A., Montgomery, J.A., Raghavachari, K., Al-Laham, M.A., Zakrzewaki, V.G., Ortiz, J.V., Foresmann, J.B., Ciolowski, J., Stefanov, B.B., Namayakkara, A., Challacombe, M., Peng, C.Y., Ayala, P.Y., Chen, W., Wong, M.W., Andres, J.L., Replogle, E.S., Gomperts, R., Martin, R.L., Fox, D.J., Binkley, J.S., Defrees, D.J., Baker, J., Stewart, J. P., Head-Gordon, M., Gonzalez, C., and Pople, J.A.. Gaussian 03, Gaussian Inc., Pittsburgh PA. 